\title{
An analytical and experimental study of heat transfer in fixed bed
}

\author{
Licínio M. Ferreira ${ }^{\text {a }}$, José A.M. Castro ${ }^{\text {a }}$, Alírio E. Rodrigues ${ }^{\text {b,* }}$ \\ a Department of Chemical Engineering, University of Coimbra, Pinhal de Marrocos, Polo II, 3030 Coimbra, Portugal \\ ${ }^{\mathrm{b}}$ Laboratory of Separation and Reaction Engineering, School of Engineering, University of Porto, 4200-465 Porto, Portugal
}

Received 29 June 2000; received in revised form 5 July 2001

\begin{abstract}
Heat transfer in a fixed-bed packed with polymeric adsorbent particles, percolated by a liquid phase flowing upward at $60{ }^{\circ} \mathrm{C}$ or downward at $20^{\circ} \mathrm{C}$, is experimentally studied in a column $9 \mathrm{~cm}$ in diameter and $1 \mathrm{~m}$ in length. The transient temperature profiles were measured axially and radially in the column. Sensitivity function calculations enabled to prove that the thermal parameters could be estimated from the experimental data and also to obtain information about the optimum location of the sensors. Experimental transient temperature responses were compared to those predicted by one-dimensional (1-D) and two-dimensional ( 2-D) pseudo-homogeneous (PH) models. By fitting the 2-D model solution to the experimental responses, values for the thermal parameter, effective radial and axial conductivities and the wall heat transfer coefficient were found. (c) 2002 Elsevier Science Ltd. All rights reserved.
\end{abstract}

\section{Introduction}

The study of heat transfer in packed beds is of considerable importance, mainly, in the analysis and design of separation units in which a fluid passing through the bed exchanges mass and heat with the particles in the presence or absence of chemical reactions. An example of such a separation is non-isothermal adsorption. In adiabatic systems with heats of adsorption and in systems of thermal swing adsorption (TSA) where the temperature changes mainly govern the separation performance, heat transfer plays an important role in the dynamic behavior of the separation process.

The parametric pumping technique is one possible mode of operation in a thermal swing process that combines the effect of the periodic temperature changes on the adsorption equilibrium with periodic changes in the flow direction. In this process, the separation is achieved by temperature variations imposed directly through the walls of the bed (direct mode) or through the feed stream (recuperative mode). Thus, it is essential

\footnotetext{
${ }^{*}$ Corresponding author. Tel.: +351-22508-1671; fax: +35122508-1674.

E-mail address: arodrig@fe.up.pt (A.E. Rodrigues).
}

to analyze these thermal effects and their interaction with the mass transfer because for significant heat loss at the wall and for large temperature gradients in the bed less separation will take place.

A general solution to the problem of predicting the process performance requires the simultaneous solution of the appropriate differential equations for heat and mass transfer. The coupling of the energy balance with the mass balance is established by the heat of adsorption term. In most liquid systems the effect of this term is negligible and thus the energy balance can be solved separately. However, this requires the knowledge of basic process parameters like, the wall heat transfer coefficient and the radial and axial thermal conductivities. The evaluation of such parameters by using empirical correlations is often subject to considerable uncertainties and, therefore, it is necessary to follow a different strategy. For instance, using the experimental dynamic responses obtained by changing the inlet fluid temperature, the parameters can be estimated by fitting the mathematical model solution to the set of experimental data. The success and relevance of this estimation depend highly on the model used to describe the behavior of the system [1]. Fixed beds are commonly described by two types of models: one-dimensional (1-D) or two-dimensional (2-D) depending on whether 


\begin{tabular}{|c|c|c|c|}
\hline \multicolumn{2}{|c|}{ Nomenclature } & \multirow{2}{*}{$\begin{array}{l}P e_{\mathrm{r}} \\
P e_{\mathrm{r}}^{*}\end{array}$} & \multirow{2}{*}{$\begin{array}{l}\text { thermal radial Peclet number (based on } \\
R_{\mathrm{c}} \text { ) } \\
\text { thermal radial Peclet number (based on } d_{\mathrm{p}} \text { ) }\end{array}$} \\
\hline$a_{\mathrm{i}}\left(z^{*}, \theta\right)$ & coefficients of the trial solution & & \\
\hline$a_{\mathrm{w}}$ & specific area of wall, $\mathrm{m}^{-1}$ & $Q$ & flowrate in the column, $\mathrm{m}^{3} \mathrm{~s}^{-1}$ \\
\hline$A$ & bed cross-sectional area, $\mathrm{m}^{2}$ & $r$ & radial coordinate, $\mathrm{m}$ \\
\hline$A_{\mathrm{b}}$ & bubble trap surface area, $\mathrm{m}^{2}$ & $r^{*}$ & normalized radial coordinate $\left(r / R_{\mathrm{c}}\right)$ \\
\hline$\left(B i_{\mathrm{f}}\right)_{\mathrm{h}}$ & fluid to solid Biot number & $R_{\mathrm{c}}$ & column radius, $\mathrm{m}$ \\
\hline$\left(B i_{\mathrm{w}}\right)_{\mathrm{h}}$ & fluid to wall Biot number & $S_{\phi_{i}}^{T}$ & sensitivity function of the dependent \\
\hline$C_{\mathrm{p}_{\mathrm{f}}}$ & heat capacity of the fluid, $\mathrm{kJ} \mathrm{kg}^{-1} \mathrm{~K}^{-1}$ & & variable $T$ with respect to the parameter \\
\hline$C_{\mathrm{p}_{\mathrm{s}}}^{\prime}$ & $\begin{array}{l}\text { heat capacity of the adsorbent, } \\
\mathrm{kJ} \mathrm{kg}^{-1} \mathrm{~K}^{-1}\end{array}$ & $t$ & $\begin{array}{l}\phi_{j} \\
\text { time, s }\end{array}$ \\
\hline$d_{\mathrm{p}}$ & particle diameter, $\mathrm{m}$ & $t_{\mathrm{f}}$ & final time, $\min$ \\
\hline$D_{\text {re }}$ & effective radial diffusivity, $\mathrm{m}^{2} \mathrm{~s}^{-1}$ & $T$ & temperature, $\mathrm{K}$ \\
\hline$g^{*}(z, r)$ & dimensionless Gram determinant & $T^{*}$ & dimensionless temperature \\
\hline$G_{\mathrm{f}}$ & fluid mass flowrate, $\mathrm{kg} \mathrm{m}^{-2} \mathrm{~s}^{-1}$ & $T_{\mathrm{a}}$ & ambient temperature, $\mathrm{K}$ \\
\hline$h$ & $\begin{array}{l}\text { overall heat transfer coefficient between } \\
\text { solid and fluid }\end{array}$ & $\begin{array}{l}T_{\mathrm{F}} \\
u_{\mathrm{i}}\end{array}$ & $\begin{array}{l}\text { feed temperature, } \mathrm{K} \\
\text { interstitial velocity, } \mathrm{m} \mathrm{s}^{-1}\end{array}$ \\
\hline$h_{k}$ & length of subinterval $k$ & $u_{\mathrm{th}}$ & thermal wave velocity, $\mathrm{m} \mathrm{s}^{-1}$ \\
\hline$h_{\mathrm{we}}$ & $\begin{array}{l}\text { overall heat transfer coefficient, } \\
\mathrm{kJ} \mathrm{m}^{-2} \mathrm{~s}^{-1} \mathrm{~K}^{-1}\end{array}$ & $\begin{array}{l}u_{k}^{*} \\
u_{k+1}^{*}\end{array}$ & $\begin{array}{l}\text { inferior limit of subinterval } k \\
\text { upper limit of subinterval } k\end{array}$ \\
\hline$H_{i}(g k)$ & $\begin{array}{l}\text { Hermite polynomials over the subinterval } \\
k\end{array}$ & $U$ & $\begin{array}{l}\text { wall heat transfer coefficient, } \\
\mathrm{kJ} \mathrm{m}^{-2} \mathrm{~s}^{-1} \mathrm{~K}^{-1}\end{array}$ \\
\hline$K_{\mathrm{ae}}$ & $\begin{array}{l}\text { effective axial thermal conductivity, } \\
\mathrm{kJ} \mathrm{m}^{-1} \mathrm{~s}^{-1} \mathrm{~K}^{-1}\end{array}$ & $\begin{array}{l}z \\
z^{*}\end{array}$ & $\begin{array}{l}\text { spatial coordinate, } \mathrm{m} \\
\text { normalized axial coordinate }(z / L)\end{array}$ \\
\hline$K_{\mathrm{f}}$ & $\begin{array}{l}\text { thermal conductivity of the fluid, } \\
\mathrm{kJ} \mathrm{m}^{-1} \mathrm{~s}^{-1} \mathrm{~K}^{-1}\end{array}$ & Gree & \\
\hline$K_{\mathrm{re}}$ & $\begin{array}{l}\text { effective radial thermal conductivity, } \\
\mathrm{kJ} \mathrm{m}^{-1} \mathrm{~s}^{-1} \mathrm{~K}^{-1}\end{array}$ & $\alpha_{\mathrm{w}}$ & $\begin{array}{l}\text { wall heat transfer coefficient, } \\
\mathrm{kJ} \mathrm{m}^{-2} \mathrm{~s}^{-1} \mathrm{~K}^{-1}\end{array}$ \\
\hline$K_{\mathrm{s}}$ & $\begin{array}{l}\text { thermal conductivity of the solid, } \\
\mathrm{kJ} \mathrm{m}^{-1} \mathrm{~s}^{-1} \mathrm{~K}^{-1}\end{array}$ & $\begin{array}{l}\xi_{\mathrm{h}} \\
\varepsilon\end{array}$ & $\begin{array}{l}\text { thermal capacity parameter } \\
\text { bed porosity }\end{array}$ \\
\hline$L$ & bed height, $\mathrm{m}$ & $\rho_{\mathrm{f}}$ & density of the fluid, $\mathrm{kg} \mathrm{m}^{-3}$ \\
\hline$N_{\mathrm{hw}}$ & number of wall heat transfer units & $\rho_{\mathrm{h}}$ & wet density of the adsorbent, $\mathrm{kg} \mathrm{m}^{-3}$ \\
\hline$N u$ & Nusselt number $\left(=h d_{\mathrm{p}} / K_{\mathrm{f}}\right)$ & $\theta$ & normalized time $(t / \tau)$ \\
\hline$P e_{\mathrm{h}}$ & thermal axial Peclet number & $\tau$ & space time, $\mathrm{s}$ \\
\hline$P e_{\mathrm{h}}^{*}$ & thermal axial Peclet number (based on $d_{\mathrm{p}}$ ) & $\tau_{\mathrm{B}}$ & residence time in the bubble trap, $\mathrm{s}$ \\
\hline
\end{tabular}

radial temperature profiles are important; moreover, these may explicitly include both phases (heterogeneous model) or only a single -homogeneous phase (homogeneous model). The heterogeneous model distinguishes between temperatures in the bulk fluid phase and that inside, or at the surface of the solid. In the homogeneous model inter- and intra-particle resistances are neglected so that the bulk fluid temperature is assumed to be the same as the solid temperature.

Let us return to the problem of estimation of the parameters. This problem is usually solved by minimizing an objective function defined as the sum squares of the differences between the experimental and predicted values. It should be noted, however, that the experimental conditions may influence the quality of the estimates, i.e., if the objective function is not sensitive to changes in a certain parameter, one would expect diffi- culties in estimating. Hence, it is crucial to assure that the set of experimental data at hand possesses the necessary information for a reliable estimation of the parameters. A possible solution to this problem is provided by the method proposed by Point et al. [8] which consists in verifying the linear independence of the sensitivity functions. This methodology requires the computation of a determinant of the so-called sensitivity matrix $g(z, r)$, similar to the Gram determinant, in various radial and axial positions of the bed. Sensitivity information may also be helpful in determining the influence of small deviations in a system parameter upon the dependent variable temperature. Sensitivity studies of this kind have been successfully applied in the analysis of chemical reactors by several authors [9-13].

In this paper the method based on the sensitivity functions is used to prove that the experimental 
conditions were suitable for the estimation of the thermal parameters. A parametric sensitivity analysis has also been carried out in order to gather information about their influence on the bed temperature. The 1-D and 2-D pseudo-homogeneous $(\mathrm{PH})$ models were used to analyze experimental transient thermal responses obtained in a packed bed by changing its inlet fluid temperature. Values of the wall coefficient, effective radial and axial conductivities were estimated for a set of operating conditions. The temperatures chosen $\left(20^{\circ} \mathrm{C}-\right.$ cold temperature and $60{ }^{\circ} \mathrm{C}$ - hot temperature) were those used in the operation of a pilot installation of thermal parametric pumping to treat effluent streams [14].

\section{Experimental description}

Transient responses were measured in the experimental set-up shown in Fig. 1. This consists of the following elements:

1. A borosilicate packed column $(1-$ Amicon G90 $\times$ 1000), non-insulated, with $0.09 \mathrm{~m}$ internal diameter and about $1 \mathrm{~m}$ height. The packing is a polymeric adsorbent (Duolite ES-861) formed by spherical particles of average diameter equal to $0.47 \mathrm{~mm}$. Five thermocouples (type K) are installed axially and radially in the column to measure the bed temperatures. Thermocouple data were collected by a computer equipped with the interface DT2805 series (Data Translation) board data acquisition system.

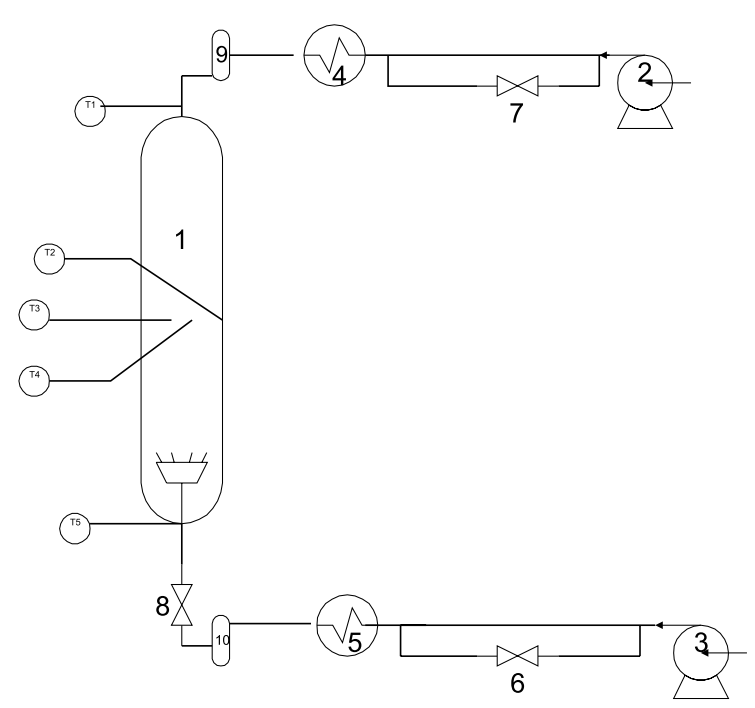

Fig. 1. Experimental apparatus. Legend: 1: Glass column G90Amicon; 2, 3: Peristaltic pumps-Watson Marlow; 4, 5: Heat exchangers; 6-8: two-ways solenoid valves; 9,10: bubble traps; T1-T5: Thermocouples (type K).
2. Two peristaltic pumps $(2,3)$ were used to percolate the solution through the column either downward (cold step input at $20^{\circ} \mathrm{C}$ ) or upward (hot step input at $60{ }^{\circ} \mathrm{C}$ ).

3. Two heat exchangers $(4,5)$ to cool and heat the fluid phase, respectively.

4. Three solenoid valves $(6,7,8)$ to direct and/or stop the flow.

5. Two bubble traps $(9,10)$ to prevent gasing out inside the fixed bed.

Two types of experiments were performed. In one, after the column is initially equilibrated at the cold temperature $\left(\approx 20^{\circ} \mathrm{C}\right)$, hot water $\left(\approx 60^{\circ} \mathrm{C}\right)$ was pumped through the column in upward flow. After a uniform temperature profile is established along the column the flow was reversed and then the other experiment started. Now, cold water at about $20^{\circ} \mathrm{C}$ was pumped in downward flow. During both experiments the temperatures were continuously recorded and collected by a data acquisition system.

\section{Analysis and modeling}

To decide whether or not a certain type of model is adequate to provide a satisfactory representation of the behavior of the experimental system, it is convenient to analyze two situations: the relative importance of external (inter-particular) - internal (intra-particular) temperature gradients and the magnitude of the radial temperature gradients in the bed.

In relation to the first situation, for the dimensionless number

$\left(B i_{\mathrm{f}}\right)_{\mathrm{h}}=\frac{N u}{6} \frac{K_{\mathrm{f}}}{K_{\mathrm{s}}} \ll 1.0$

it is possible to conclude that the external temperature gradient is much greater than the temperature gradient within the particle. As a consequence, the temperature can be considered uniform throughout the adsorbent particle and this is in fact a good approximation for most systems. Regarding the interparticular gradient, the heat transfer coefficient between particle and fluid $\left(h_{\mathrm{wf}}\right)$ should be estimated in order to analyze the contribution of that gradient in the overall heat transfer. Most of the studies reported in the literature advocate the use of correlations that express $h_{\mathrm{wf}}$ in terms of the flow conditions, i.e. the Reynolds number. For relatively large particles and high fluid velocities, the contribution of the interparticular resistance has been found to be small and this means that the pseudo-homogeneous formulation can be a good representation of the system.

The second situation, which is concerned with the magnitude of the radial temperature profiles, raises the question if the pseudo-homogeneous model should be 
extended to the 2-D case. In order to derive a semiquantitative criterion for the existence of radial temperature gradients, consider the following two regions for radial heat transfer in the bed - one, a fictitious film near the wall and the other, the core of the bed. Assume also that the film thickness is small compared with the bed radius and that any variation in temperature between the edge of the film and the center of the bed is linear. Thus, the total resistance to heat flow in the radial direction can be considered as the sum of two thermal resistances: the resistance to heat flow at the wall that is proportional to $1 / \alpha_{w}$ and the resistance to radial heat flow through the bed that is proportional to $R_{\mathrm{c}} /\left(D_{\mathrm{re}} \rho_{\mathrm{f}} C_{\mathrm{p}_{\mathrm{f}}}\right)$. The ratio of these resistances gives a good indication of the relative importance of the radial temperature gradients. The equivalence between the 2- and 1-D pseudo-homogeneous models was analyzed by Dixon [2,3]. The ability of these models in describing transient responses of a packed bed has been shown in numerous works [4-7].

Some difficulties may occur in applying results from a laboratory or pilot plant scale to a commercial unit. In a column of commercial size large volumes of solution are percolated and one difficulty for a parametric pumping process operating in direct mode is the problem of transferring heat into and out of the column. As the bed diameter increases the heating and cooling times increase and this means that exceedingly long cycles would be required. It should also be noted that for a column of larger diameter the non-isothermal effects will be more pronounced leading to a greater interference on the adsorption wave pattern in the bed. The practical implication of this is that the design and scale-up of TSA processes, often based on simple equations in which is assumed a constant-pattern behavior for the propagation of concentration waves inside the bed, can lead to erroneous results. Thus, it is essential to describe the problem with a model that takes into account all the transfer mechanisms in order to correctly interpret the experimental results and to enable the scaling-up to the industrial conditions.

\subsection{The one-dimensional pseudo-homogeneous model (1- $D, P H)$}

The simplest model for the analysis of heat transfer in a fixed bed is the 1-D pseudo-homogeneous model which combines the resistance of the bed (effective radial conduction) and the wall resistance (wall heat transfer coefficient) into a single overall heat transfer coefficient related to the bed radial average temperature.

A differential energy balance for the process described above leads to the following dimensionless equation:

$$
\begin{aligned}
& \frac{1}{P e_{\mathrm{h}}} \frac{\partial^{2} T^{*}\left(z^{*}, \theta\right)}{\partial z^{* 2}}-\frac{\partial T^{*}\left(z^{*}, \theta\right)}{\partial z^{*}} \\
& \quad=\left(1+\xi_{\mathrm{h}}\right) \frac{\partial T^{*}\left(z^{*}, \theta\right)}{\partial \theta}-N_{\mathrm{hw}} T^{*}\left(z^{*}, \theta\right)=0,
\end{aligned}
$$

where $T^{*}=\left(T-T_{\mathrm{a}}\right) /\left(T_{\mathrm{F}}-T_{\mathrm{a}}\right)$ is the dimensionless temperature; $z^{*}=z / L$ is the normalized axial coordinate and $\theta=t / \tau(\tau=\varepsilon L / u)$ is the dimensionless time. The terms on the left-hand side of this equation represent the heat transfer in the axial direction by effective conduction and convection, while the terms on the right-hand side describe the rate of heat accumulation and the rate of heat transfer to the environment.

The boundary and initial conditions are:

$$
\begin{array}{ll}
z^{*}=0: & T^{*}\left(z^{*}, \theta\right)=1, \\
z^{*}=1: & \frac{\partial T^{*}\left(z^{*}, \theta\right)}{\partial z^{*}}=0, \\
\theta=0:= & T^{*}\left(z^{*}, 0\right)=T_{0}^{*} \quad\left(z^{*}>0\right), \\
& T^{*}(0,0)=1 \quad\left(z^{*}=0\right)
\end{array}
$$

and the dimensionless parameters are defined as follows:

Axial thermal Peclet number,

$P e_{\mathrm{h}}=\frac{G_{\mathrm{f}} C_{\mathrm{p}_{\mathrm{f}}} L}{K_{\mathrm{ae}}}$.

Number of wall heat transfer units,

$N_{\mathrm{hw}}=\frac{h_{\mathrm{we}} a_{\mathrm{w}} L}{G_{\mathrm{f}} C_{\mathrm{p}_{\mathrm{f}}}}$.

Thermal capacity factor,

$\xi_{\mathrm{h}}=\frac{(1-\varepsilon) \rho_{\mathrm{h}} C_{\mathrm{p}_{\mathrm{s}}}^{\prime}}{\varepsilon \rho_{\mathrm{f}} C_{\mathrm{p}_{\mathrm{f}}}}$.

The thermal capacity parameter $\xi_{\mathrm{h}}$ was evaluated on the basis of the heat capacity ratios of both phases. The value of $0.72 \mathrm{~kg}$ water $/ \mathrm{kg}$ wet resin (water content of the wet resin) obtained experimentally was used to calculate $C_{\mathrm{p}_{\mathrm{s}}}^{\prime}\left(=3.42 \mathrm{~kJ} \mathrm{~kg}_{\text {wet resin }}^{-1} \mathrm{~K}^{-1}\right)$. To estimate the number of units of wall heat transfer $N_{\text {hw }}$, the overall heat transfer coefficient was calculated by fitting the experimental data at the top of the column with the analytical steady-state solution $\left(T^{*}\left(z^{*}\right)=\mathrm{e}^{-N_{\mathrm{hw}} z^{*}}\right)$ of the model (Eq. (1)) and assuming plug flow for the fluid phase.

Fig. 2 compares histories of experimental (Run 1) and simulated exit temperatures for several values of the thermal Peclet number. For larger values of this parameter, i.e., decreasing thermal axial dispersion effects, the curves tend to the solution of the model in which the axial dispersive effect can be neglected (plug flow case). The experimental conditions for this run are shown in Table 1.

Since there are two bubble traps (small cylindrical reservoirs) located at the two ends of the column, the hot 


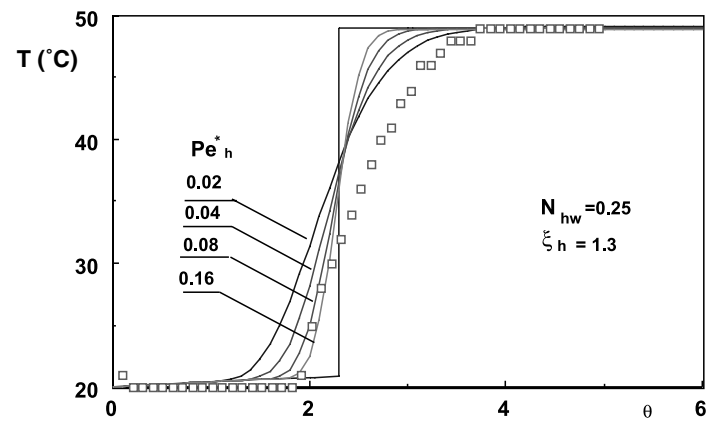

Fig. 2. Effect of the thermal axial Peclet number on history of fluid temperatures at the outlet of the column. Full (axial dispersed plug flow) and dashed (plug flow) lines are simulated results with the 1-D pseudo-homogeneous model.

feed is perfectly mixed with cold liquid inside the reservoir before entering into the column. As a result of this, the time evolution of the bed inlet temperature in $z^{*}=0$ is not a pure Heaviside function (step) as stated by Eq. (2) but should take the form of an exponential input,

$T^{*}(t)=\frac{\beta_{2}}{\beta_{1}}\left[1-\exp \left(-\beta_{1} t / \tau_{\mathrm{B}}\right)\right]$,

where

$$
\begin{aligned}
& \beta_{1}=1+U A_{\mathrm{b}} /\left(Q \rho_{\mathrm{f}} C_{\mathrm{p}_{\mathrm{f}}}\right) ; \quad \beta_{2}=1+U A_{\mathrm{b}} /\left(Q \rho_{\mathrm{f}} C_{\mathrm{p}_{\mathrm{f}}}\right) \delta \\
& \text { and } \delta=\left(T_{\mathrm{a}}-T_{\mathrm{Fo}}\right) /\left(T_{\mathrm{F}}^{\prime}-T_{\mathrm{Fo}}\right)
\end{aligned}
$$

The parameters $\beta_{1}$ and $\beta_{2}$ were evaluated taking the limit of Eq. (6) as $t \rightarrow \infty$ and with $T_{\mathrm{F}}^{\prime}$ (feed temperature before entering into bubble trap) $=57{ }^{\circ} \mathrm{C}$ and $U A_{\mathrm{b}}$ (wall heat transfer coefficient $x$ heat transfer area) $=0.46 \mathrm{~J} \mathrm{~s}^{-1} \mathrm{~K}^{-1}$. The residence time in bubble trap, $\tau_{\mathrm{B}}$, was found to be $\tau_{\mathrm{B}}=2 \mathrm{~min}$.

When this modified boundary condition takes the place of Eq. (2) in the model equations (1)-(5) the predicted dynamic temperature profile at the exit of the column differs from those shown in Fig. 2. In particular for the case of $P e_{\mathrm{h}}^{*}=0.04, N_{\mathrm{hw}}=0.25$ and $\xi_{\mathrm{h}}=1.3$ this simulated temperature profile is now much closer to the experimental temperature history, as highlighted in Fig. 3.

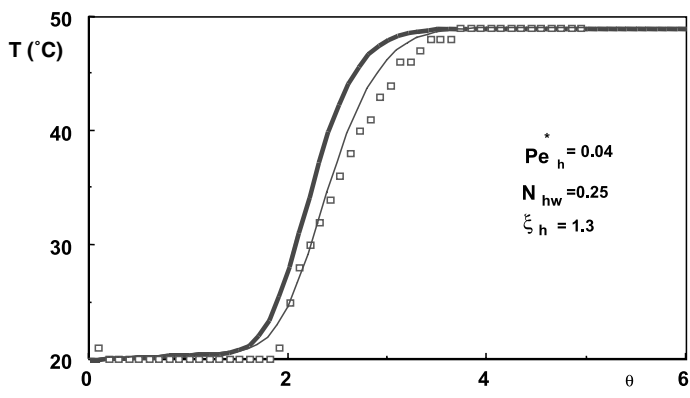

Fig. 3. Histories of experimental and simulated fluid temperatures at the outlet of the column; Full lines are simulated results with the 1-D pseudo-homogeneous model; step (thick line) and exponential ( - inputs.

\subsection{The two-dimensional pseudo-homogeneous model (2- $D, P H)$}

When the radial temperature profiles cannot be neglected a 2-D model of the temperature distribution in the fixed bed is necessary for a complete description of its thermal dynamic behavior. The corresponding dimensionless model equations are now as follows:

$$
\begin{aligned}
\frac{1}{P e_{\mathrm{h}}} & \frac{\partial^{2} T^{*}\left(z^{*}, r^{*}, \theta\right)}{\partial z^{* 2}} \pm \frac{\partial T^{*}\left(z^{*}, r^{*}, \theta\right)}{\partial z^{*}} \\
= & \left(1+\xi_{\mathrm{h}}\right) \frac{\partial T^{*}\left(z^{*}, r^{*}, \theta\right)}{\partial \theta} \\
& -\frac{L}{R_{\mathrm{c}} P e_{\mathrm{r}}}\left[\frac{\partial^{2} T^{*}\left(z^{*}, r^{*}, \theta\right)}{\partial r^{* 2}}+\frac{1}{r^{*}} \frac{\partial T^{*}\left(z^{*}, r^{*}, \theta\right)}{\partial r^{*}}\right]
\end{aligned}
$$

$(-)$ : upward flow; (+): downward flow

with the boundary conditions

$$
\begin{array}{ll}
z^{*}=0: & T^{*}(\theta)=\frac{\beta_{2}}{\beta_{1}}\left[1-\exp \left(-\beta_{1} \theta \tau / \tau_{\mathrm{B}}\right)\right], \\
z^{*}=1: & \frac{\partial T^{*}\left(z^{*}, r^{*}, \theta\right)}{\partial z^{*}}=0, \\
r^{*}=0: & \frac{\partial T^{*}\left(z^{*}, r^{*}, \theta\right)}{\partial r^{*}}=0,
\end{array}
$$

\begin{tabular}{|c|c|c|c|c|c|c|c|}
\hline Run/flow direction & $T_{\mathrm{a}}\left({ }^{\circ} \mathrm{C}\right)$ & $T_{\mathrm{F}}^{\prime}\left({ }^{\circ} \mathrm{C}\right)$ & $T_{\text {Fo }}\left({ }^{\circ} \mathrm{C}\right)$ & $\begin{array}{l}Q \times 10^{6} \\
\left(\mathrm{~m}^{3} \min ^{-1}\right)\end{array}$ & $\tau(\min )$ & $\tau_{\mathrm{B}}(\min )$ & $\begin{array}{l}U A \times 10^{4} \\
\left(\mathrm{~kJ} \mathrm{~s}^{-1} \mathrm{~K}^{-1}\right)\end{array}$ \\
\hline 1/upward & 24 & 57 & 20 & 220 & 9.9 & 2.0 & 4.6 \\
\hline 2/upward & 22 & 62 & 21 & 192 & 11.3 & 2.0 & 4.6 \\
\hline 3/downward & 24 & 14 & 56 & 275 & 7.9 & 0.9 & 5.0 \\
\hline 4/downward & 25 & 18 & 60 & 240 & 9.1 & 2.5 & 5.0 \\
\hline
\end{tabular}

Table 1

Experimental conditions for the thermal experiments 
$r^{*}=1: \quad-\frac{\partial T^{*}\left(z^{*}, r^{*}, \theta\right)}{\partial r^{*}}=\left(B i_{\mathrm{w}}\right)_{\mathrm{h}}\left[T^{*}\left(1, z^{*}, \theta\right)-\delta\right]$

and initial conditions

$\theta=0: \quad T^{*}\left(z^{*}, r^{*}, 0\right)=T_{0}^{*}\left(z^{*}\right) \quad\left(z^{*}, r^{*}>0\right)$.

In this model there are two additional parameters:

Radial thermal Peclet number,

$P e_{\mathrm{r}}=\frac{G_{\mathrm{f}} C_{\mathrm{p}_{\mathrm{f}}} R_{\mathrm{c}}}{K_{\mathrm{re}}}$.

Thermal Biot number,

$\left(B i_{\mathrm{w}}\right)_{\mathrm{h}}=\frac{\alpha_{\mathrm{w}} R_{\mathrm{c}}}{K_{\mathrm{re}}}$.

The terms on the left-hand side of Eq. (7) are analogous to those in Eq. (1) while the terms on the righthand side describe the rates of heat accumulation and of heat transfer in the radial direction of the bed by conduction and by exchange with the column wall.

\subsubsection{Numerical method}

The model equations presented above are of parabolic partial differential type, in two spatial dimensions $r^{*}$ and $z^{*}$ and in time $\theta$. For their numerical solution, the equations are firstly transformed into a set of $2 N E$ one space dimension $P D E$ 's by discretizing the normalized radial coordinate $r^{*}$ in $N E$ subintervals. An approximate solution in each subinterval $k$ can then be represented by [15]:

$T^{*}\left(z^{*}, g_{k}, \theta\right)=\sum_{i=1}^{4} H_{i}\left(g_{k}\right) a_{i+2 k-2}\left(z^{*}, \theta\right)$

with $k=1,2,3, \ldots, N E$ subintervals. $H_{i}\left(g_{k}\right)$ are the cubic Hermite polynomials, $a_{i+2 k-2}$ are coefficients to be determined and $g_{k}$ is the normalized radial coordinate for the subinterval $k$,

$g_{k}=\frac{r^{*}-r_{k}^{*}}{h_{k}} \quad$ with $h_{k}=r_{k+1}^{*}-r_{k}^{*}$.

Thereafter, the resulting $2 N E P D E$ 's are solved by a software package - PDASAC [16], based on finite element collocation; 19 subintervals in the axial direction and 6 subintervals in the bed radial direction were used in the numerical solution.

\subsection{Sensitivity studies}

In this section a dynamic parametric sensitivity analysis is carried out with the purpose of investigating the effect of the thermal parameters on the temperature dynamic behavior of the system. In addition to this, the sensitivity functions can provide relevant information for determining optimum experimental conditions such as those reported by Point et al. [8]. The following normalized sensitivity functions were adopted:
$S_{\phi_{j}}^{T}(z, r, t)=\left(\frac{\partial T(z, r, t)}{\partial \phi_{j}}\right) \phi_{j}$.

An important feature of the above-mentioned package PDASAC is that it simultaneously computes such sensitivities with the solution of the model equations.

The influence of the thermal and operating parameters on the fixed-bed transient temperature responses is now examined. The thermal parameters, $K_{\mathrm{ae}}$, $K_{\text {re }}$ and $\alpha_{w}$, are unknowns to be estimated from the experimental data. Among the operating parameters, only $Q$ and $L$, can be changed freely since those related to the fluid, particle and bed are fixed.

The numerical values used for the thermal parameters are:

$K_{\mathrm{ae}}=23.11 \mathrm{~J} \mathrm{~m}^{-1} \mathrm{~s}^{-1} \mathrm{~K}^{-1}$,

$K_{\mathrm{re}}=0.15 \mathrm{~J} \mathrm{~m}^{-1} \mathrm{~s}^{-1} \mathrm{~K}^{-1}$,

$\alpha_{\mathrm{w}}=24.83 \mathrm{~J} \mathrm{~m}^{-2} \mathrm{~s}^{-1} \mathrm{~K}^{-1}$.

The effective axial conductivity $K_{\mathrm{ae}}$ was obtained from the value of $P e_{\mathrm{h}}^{*}, P e_{\mathrm{h}}^{*}=0.04$, estimated in the previous study with the 1-D model. Based on a value of $P e_{\mathrm{r}}^{*}=7.5$ (typical values of $P_{\mathrm{er}}^{*}$ are in the range 5-10, [17]), one is able to estimate the effective radial conductivity $K_{\text {re }}$.

Calculation of $\alpha_{w}$ can be achieved by fitting the analytical steady-state solution of the 2-D model [18] to the experimental response at the outlet of the column.

$\bar{T}=\frac{4\left(B i_{\mathrm{w}}\right)_{\mathrm{h}}^{2} \exp \left(\rho_{1} z^{*}\right)}{\left[\left(B i_{\mathrm{w}}\right)_{\mathrm{h}}^{2}+\beta_{1}^{2}\right]\left(1-\rho_{1} / P e_{\mathrm{h}}^{*}\right) \beta_{1}^{2}}$,

where $\bar{T}\left(=\left(T\left(z^{*}, r^{*}\right)-T_{\mathrm{a}}\right) /\left(T_{\mathrm{F}}-T_{\mathrm{a}}\right)\right)$ is the normalized radial average temperature, $z^{*}$ and $r^{*}$ are the dimensionless axial and radial positions measured in terms of $d_{\mathrm{p}}$, respectively, $\beta_{1}$ is the smallest positive root of the equation $\beta J_{1}(\beta)-\left(B i_{\mathrm{w}}\right)_{\mathrm{h}} J_{0}(\beta)=0$ (with $J_{0}$ and $J_{1}$ being Bessel functions of the first kind of order zero and one, respectively) and $\rho_{1}$ is the negative root of

$\rho^{2}-P e_{\mathrm{h}}^{*}-\frac{4 P e_{\mathrm{h}}^{*} \beta_{1}}{\left(2 R_{\mathrm{c}} / d_{\mathrm{p}}\right)^{2} P e_{\mathrm{r}}^{*}}=0$

associated with $\beta_{1}$. To evaluate the Biot number $\left(B i_{\mathrm{w}}\right)_{\mathrm{h}}$ the following formula $\left(1 / h_{\mathrm{w}}=1 / \alpha_{\mathrm{w}}+R_{\mathrm{c}} / \beta K_{\mathrm{re}}\right)$, derived from the equivalence between the 1-D and 2-D pseudohomogeneous models, was adopted and using $h_{\mathrm{we}}=15.83 \mathrm{~J} \mathrm{~m}^{-2} \mathrm{~K}^{-1} \mathrm{~s}^{-1}$ (from the study with the 1-D model) a value of $\beta=9$ was found.

With the thermal parameters calculated previously and for Run 1, with the operating conditions shown in Table 1, the numerical solution of the 2-D model leads to the radial and axial transient temperature responses. As time increases, the radial temperature profiles exhibit a decreasing variation only in the vicinity of the wall, at a distance of $16 d_{\mathrm{p}}\left(R_{\mathrm{c}}=96 d_{\mathrm{p}}\right)$. This means that in ap- 


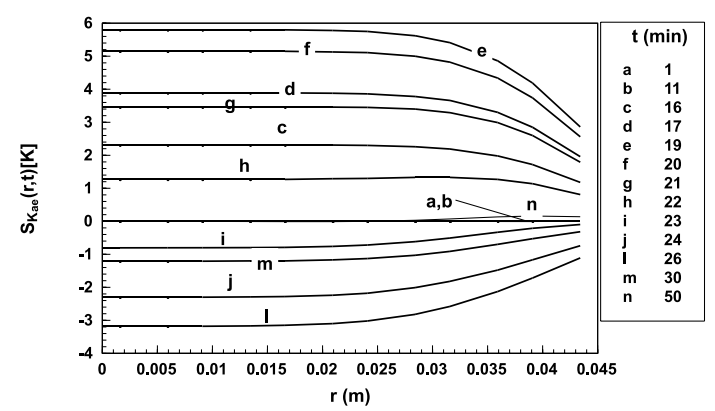

Fig. 4. Radial sensitivity profiles with respect to the effective axial conductivity, $S_{\text {Kae }}(r, t)$, for various times, at the outlet of the column.

proximately $83 \%$ of the bed radius the profile is almost flat, suggesting that most of the thermal resistance occurs at the wall.

With respect to the axial fluid temperature profiles, at different times, there is a propagation of a dispersive thermal wave inside the bed. This dispersive behavior is typical in liquid-solid thermal systems, where the axial dispersion is the only contribution to the spreading of the thermal wave since the variations in physical properties were neglected. With regard to the process dynamics, the equilibrium theory allows the calculation of the average temperature wave velocity $\left(u_{\mathrm{th}}=\right.$ $\left.u_{\mathrm{i}} /\left(1+\xi_{\mathrm{h}}\right)\right)$ that is inversely proportional to the thermal capacity parameter. Using $\xi_{\mathrm{h}}=1.3$ one obtains $u_{\mathrm{th}}=0.435 u_{\mathrm{i}}$. Considering $u_{\mathrm{i}}=0.143 \mathrm{~cm} \mathrm{~s}^{-1} \quad(=Q / A$, $\left.Q=3.67 \times 10^{-6} \mathrm{~cm}^{3} \mathrm{~s}^{-1}, \varepsilon=0.4, A=0.0064 \mathrm{~m}^{2}\right), \quad$ the time required for the thermal wave to pass through the bed $(L=0.85 \mathrm{~m})$ is around $23 \mathrm{~min}$.

\subsubsection{Steady-state sensitivity functions from 1-D, PH model}

In order to understand how sensitivity analysis can be used to improve the estimation of the thermal parameters in a steady state experimental program the corresponding sensitivity functions of the 1-D pseudohomogeneous model are studied. These correspond to the spatial profiles of the sensitivity $S_{\phi_{j}}^{T}$ along the coordinate $z$ when the temperature of the bed is stabilized. To calculate the values of $S_{\phi_{j}}^{T}$, the steady-state analytical solution of the 1-D model was used,

$$
T^{*}\left(z^{*}\right)=\exp \left\{\frac{Q \rho_{\mathrm{f}} C_{\mathrm{p}_{\mathrm{f}}} L}{2 K_{\mathrm{ae}} A}\left[1-\sqrt{1+\frac{8 h_{\mathrm{we}} K_{\mathrm{ae}} A^{2}}{R_{\mathrm{c}}\left(\rho_{\mathrm{f}} C_{\mathrm{p}_{\mathrm{f}}}\right)^{2} Q^{2}}} z^{*}\right\}\right.
$$

The obtained sensitivity functions showed that parameters $K_{\mathrm{ae}}$ and $Q$ influence positively the temperature $T(z)$ whilst parameters $h_{\mathrm{we}}$ and $L$ have an inverse effect. Along the spatial coordinate, the sensitivity increases or decreases continuously reaching either the largest or the smallest value at the outlet of the column. The effects of
$Q, L$ and $h_{\mathrm{we}}$ on the temperature are similar, i.e., the magnitude of the extreme values of the respective sensitivities are about equal. These parameters strongly influence the temperature while a very weak dependence is observed with respect to $K_{\text {ae }}$. From these results one can conclude that if the purpose of the estimation is to obtain values for $h_{\text {we }}$, then the best position to locate the temperature sensor is at the exit of the column where the sensitivity is at its maximum. These results also confirm the extreme difficulty or even the impossibility of estimating dispersion coefficients in distributed systems with steady-state experiments.

\subsubsection{Transient sensitivity functions from 2-D, PH model}

In the next study, the transient 2-D sensitivity functions $S_{\phi_{i}}^{T}(z, r, t)$, at outlet of the column, are investigated. Fig. 4 shows the radial sensitivity profiles for the parameter $K_{\mathrm{ae}}$. It is worth mentioning that the sensitivity starts to change positively from $t \approx 11$ min reaching a maximum value at about $t \approx 19 \mathrm{~min}$; thereafter, it decreases, changing from positive to negative, passing by a minimum at $t=26 \min$ and reaching a very low absolute value at steady-state. Along the radial direction, it is clear that the absolute value of the sensitivity is always smaller than in the core of the bed thus suggesting that the inner temperatures provide better information for estimating the effective axial thermal conductivity of the bed, $K_{\mathrm{re}}$.

Figs. 5 and 6 illustrate the radial and axial sensitivity profiles with respect to the parameter $K_{\text {re }}$, respectively. In Fig. 5 it is visible that in the inner radial half of the bed $\left(R_{\mathrm{c}} / 2\right)$ the sensitivity remains practically constant; between $R_{\mathrm{c}} / 2$ and the tube wall the sensitivity starts to increase slightly during a short period then it decreases quickly taking more negative values as the system approaches steady-state. With respect to the axial sensitivity profiles a similar trend exists as can be observed in Fig. 6, suggesting that the zones located near the wall and at the exit are the most sensitive to the effective radial thermal conductivity of the 2-D pseudo-homogeneous model.

\subsection{Optimum sensor locations}

We have already mentioned in Section 1 that the sensitivity functions can be useful in determining the optimum experimental conditions, i.e., sensor locations and input signals.

The method that follows has been successfully applied to several process models and consists of an evaluation of the linear independence between the sensitivity functions. These are linearly dependent if and only if the Gram determinant $g(z, r)$ vanishes, i.e., [8]

$$
\left|\begin{array}{cccc}
\int_{0}^{\mathrm{t}_{\mathrm{f}}}\left(S_{\phi_{1}}^{T}(t, z, r)\right)^{2} \mathrm{~d} t & \cdots & \int_{0}^{\mathrm{t}_{\mathrm{f}}} S_{\phi_{1}}^{T}(t, z, r) S_{\phi_{\mathrm{p}}}^{T}(t, z, r) \mathrm{d} t \\
\cdots & \cdots & \cdots & \int_{0}^{\mathrm{t}_{\mathrm{f}}} S_{\phi_{\mathrm{p}}}^{T}(t, z, r) S_{\phi_{1}}^{T}(t, z, r) \mathrm{d} t \\
\cdots & & \int_{0}^{\mathrm{t}_{\mathrm{f}}}\left(S_{\phi_{\mathrm{p}}}^{T}(t, z, r)\right)^{2} \mathrm{~d} t
\end{array}\right|=0
$$




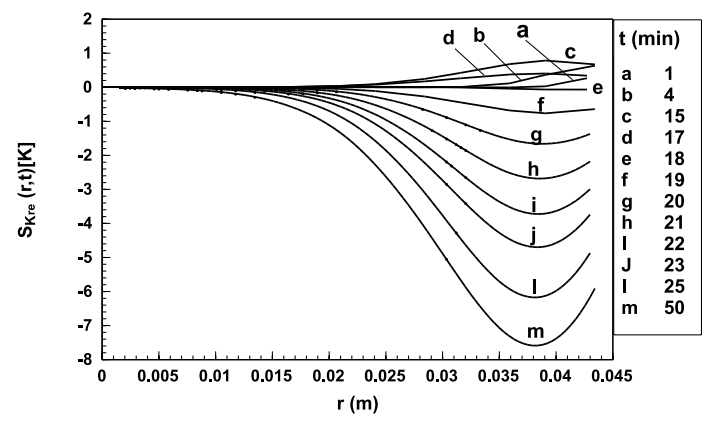

Fig. 5. Radial sensitivity profiles with respect to the effective radial conductivity, $S_{\mathrm{Kre}}(r, t)$, for various time, at the outlet of the column.

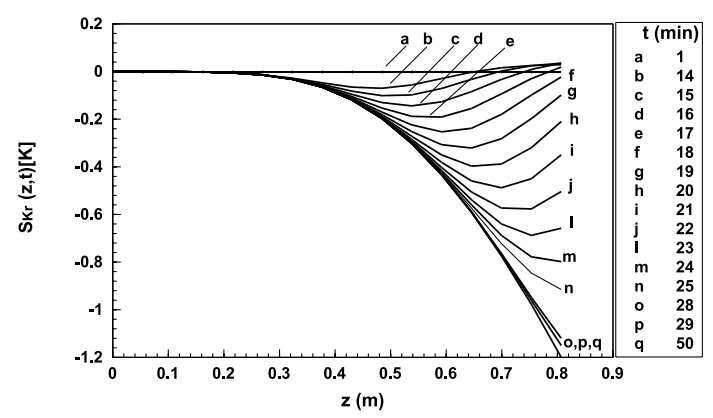

Fig. 6. Axial sensitivity profiles with respect to the effective radial conductivity, $S_{\mathrm{Kre}}(r, t)$, for various times, at $r=R_{\mathrm{c}} / 2$.

In normalized form the Gram determinant can be expressed as

$g^{*}(z, r)=\frac{g(z, r)}{\left(t_{\mathrm{f}}^{2} T_{\mathrm{F}}^{4}\right)}$

where $t_{\mathrm{f}}(=50 \mathrm{~min})$ is the final time for each run and $T_{\mathrm{F}}(=330.2 \mathrm{~K})$ is the feed temperature.

The main goal of this study is to determine the experimental conditions that lead to the linear independence of all the sensitivity functions and to ensure that the sensors are located in the spatial region where the sensitivity takes the largest absolute values.

To calculate the Gram determinant a code based on Gaussian elimination by partial pivoting was used with the integrals evaluated by a numerical quadrature technique. The results are presented in Fig. 7, where the function $g^{*}(z, r)$ is plotted against the spatial coordinate. Here, the sensitivity functions with respect to the thermal parameters $K_{\text {ae }}$ and $K_{\text {re }}$ were used in order to determine the optimal sensor location. From this Figure, it can be observed in the following: (i) for the radial points located between 0 and $0.8 R_{\mathrm{c}}, g^{*}(z, r)$ grows continuously along the axial coordinate; (ii) in the re-

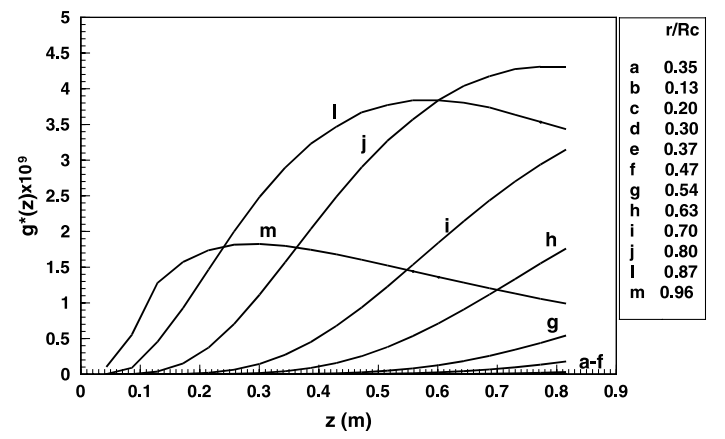

Fig. 7. Axial profiles of the function $g^{*}(z, r)$ for various radial positions.

maining zone, next to the wall, $g^{*}(z, r)$ grows to a maximum and then decreases towards the top of the column. As a result of this, any sensor located along the axial coordinate and in a radial position next to the wall, assures linear independence of the sensitivity functions $-g(z, r) \neq 0$ and this suggesting that the parameters can be estimated from the experimental data. In opposition to this, for radial distances lower than $R_{\mathrm{c}} / 2$, i.e., in the first radial half of the column the values of $g^{*}(z, r)=0$ which means that the parameters cannot be estimated from information provided by sensors located in this zone. The optimal sensor location therefore corresponds to a maximum of $g^{*}(z, r)$, at the outlet of the column, in a radial position approximately equal to $80 \% R_{\mathrm{c}}$.

Fig. 8 shows the effect of the flowrate on the radially averaged function $g^{*}(z)$ along the spatial coordinate $z$. At low flowrates, a maximum value occurs for $g^{*}(z)$ and as the flowrate decreases the $g^{*}(z)_{\max }$ region moves towards the inlet of the column. At high flowrates, any sensor location in the bed is critical, i.e., $g^{*}(z)$ is small along the spatial coordinate and the sensitivity functions become linearly dependent.

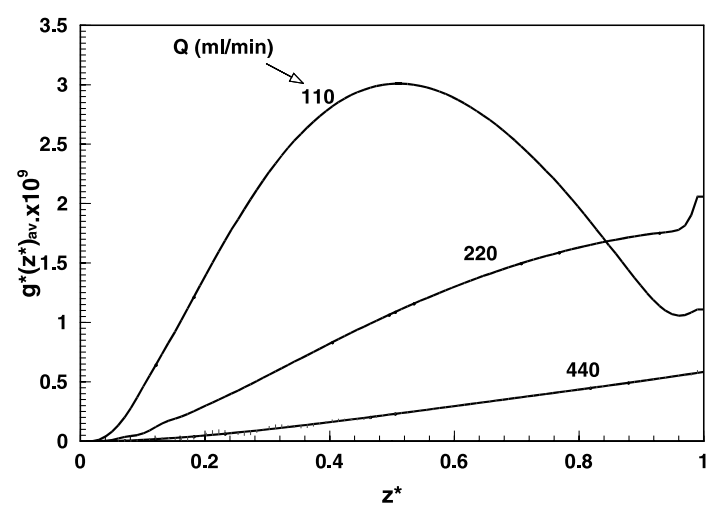

Fig. 8. Effect of the flowrate on the axial profiles of the radial average function $g^{*}(z, r)$. 

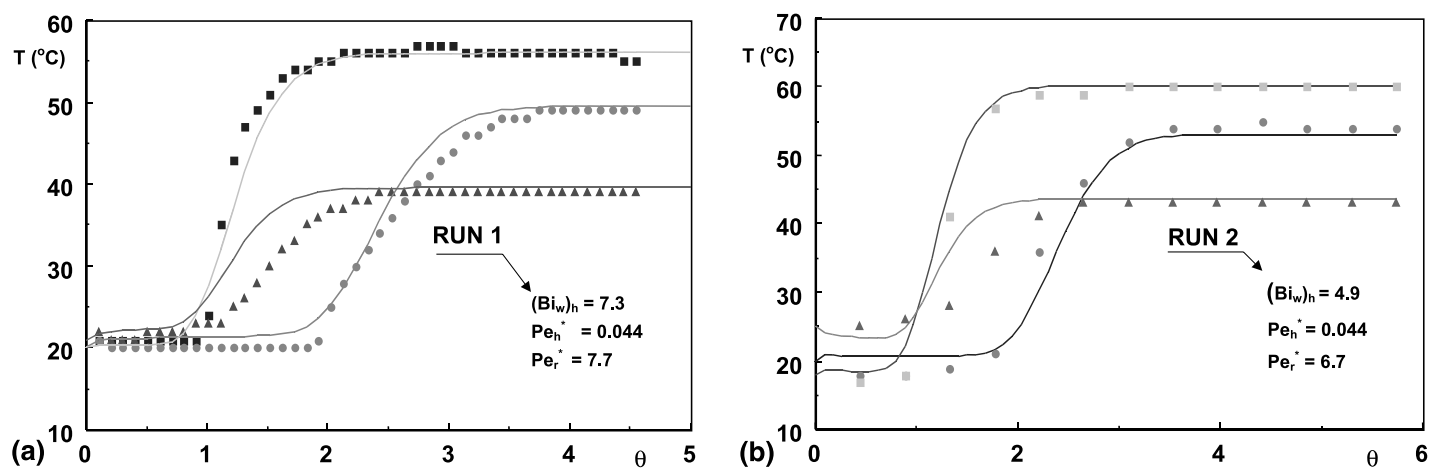

Fig. 9. (a) Histories of experimental and simulated temperatures with the 2-D model for Run 1 (upward flow at $Q=220 \mathrm{ml} \mathrm{min}^{-1}$ ). Average top $(\mathbf{O})-z^{*}=1$; middle $(\boldsymbol{\square})-z^{*}=0.5, r^{*}=0$ and wall $(\boldsymbol{\Delta})-z^{*}=0.5, r^{*}=1$ and (b) Histories of experimental and sim-

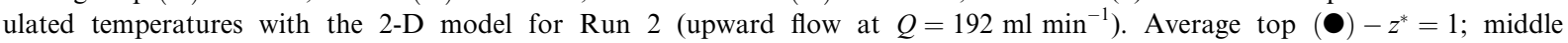
(ם) $-z^{*}=0.5, r^{*}=0$ and wall $(\boldsymbol{\Delta})-z^{*}=0.5, r^{*}=1$.

\section{Simulation of experimental results}

All the experiments were carried out according to the procedure described in Section 1, in which transient temperatures in the bed were monitored at various axial and radial locations during either the heating or the cooling periods. Experimental conditions are summarized in Table 1. Figs. 9-11 compare experimental temperature profiles with those obtained with the 2-D pseudo-homogeneous model. In Figs. 9(a) and (b) the fluid temperatures are plotted against the normalized time, $\theta$, for a positive step change in inlet fluid temperature and for two different flowrates. Similar results but for a negative step change in the inlet temperature causing the cooling of the bed are shown in Figs. 10(a) and (b). It is clear that the thermal dynamic response of a packed bed is sensitive to the flowrate variation and is reflected in the tem- perature profiles when the system reaches its thermal steady-state. The effect of the flowrate should be mainly associated to the wall energy loss; thus, higher energy loss is observed at lower flowrates and as a consequence higher differences between the temperature of the top and bottom $\Delta T_{\mathrm{b}}$, at steady state, are registered. This can be seen by comparing Runs 1 and 2 (Figs. 9(a) and (b)), when the flowrate decreased

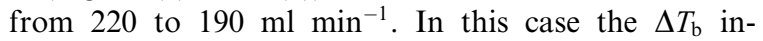
creases from $7-8$ to $10-11{ }^{\circ} \mathrm{C}$. A similar comparison can be made, along the radius, with respect to the temperatures at the center and wall for a given axial position.

Fig. 11 exhibits fluid radial temperature profiles that are almost flat up to at radial distance approximately equal to $R_{\mathrm{c}} / 2$ from the column center. The agreement between experimental and simulated results is good in all cases, except for the transient temperatures measured at
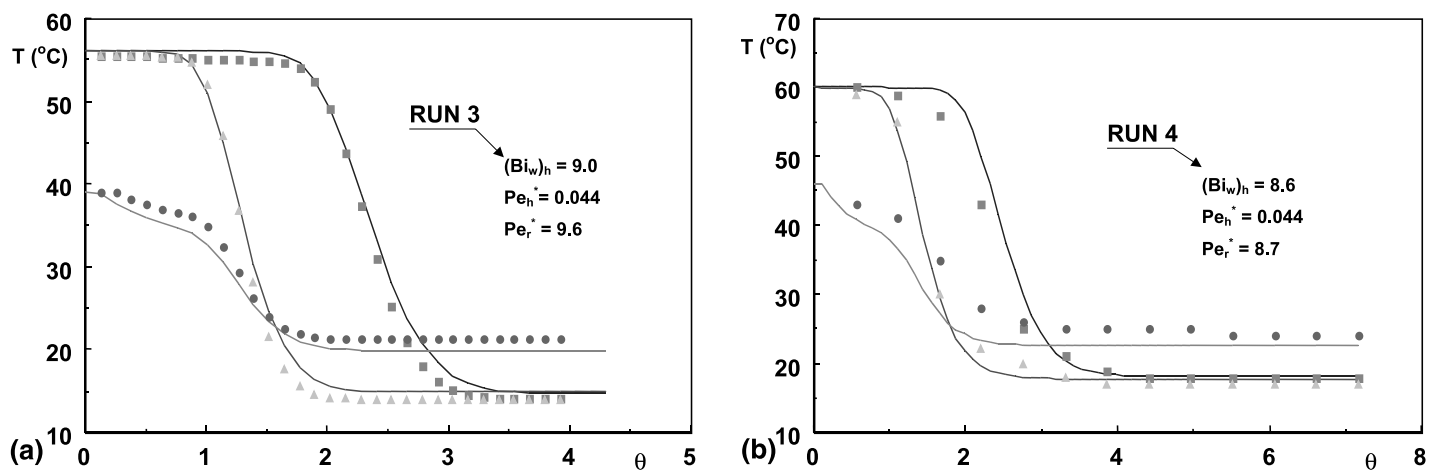

Fig. 10. (a) Histories of experimental and simulated temperatures with the 2-D model for Run 3 (downward flow at $275 \mathrm{ml} \mathrm{min}^{-1}$ ). Bottom $(\mathbf{\square})-z^{*}=1$; middle $(\Delta)-z^{*}=0.5, r^{*}=0$ and wall $(\mathbf{O})-z^{*}=0.5, r^{*}=1$. (b) Histories of experimental and simulated temperatures with the 2-D model for Run 4 (downward flow at $Q=240 \mathrm{ml} \mathrm{min}{ }^{-1}$ ). Bottom $(\mathbf{\square})-z^{*}=1$; middle $(\triangle)-z^{*}=0.5$, $r^{*}=0$ and wall $(\mathbf{O})-z^{*}=0.5, r^{*}=1$. 


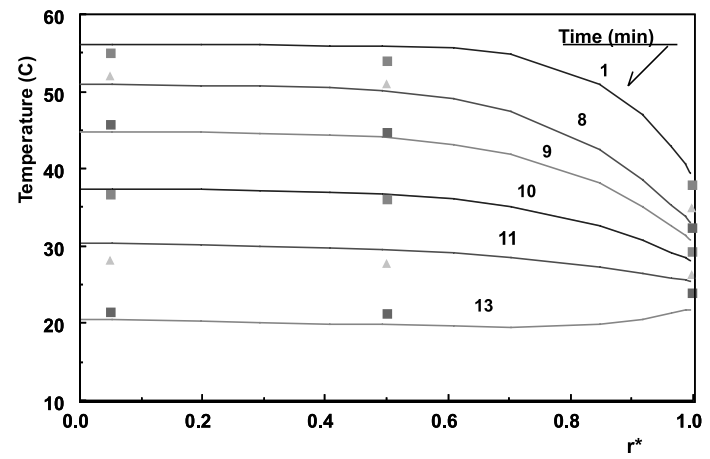

Fig. 11. Experimental and simulated radial temperatures profiles for various times, at the middle of the column $\left(z^{*}=0.5\right)$, with respect to the Run 3.

Table 2

Thermal coefficient values used in the simulations

\begin{tabular}{llll}
\hline Run & $\begin{array}{l}K_{\mathrm{ae}} \\
\left(\mathrm{J} \mathrm{m}^{-1} \mathrm{~s}^{-1} \mathrm{~K}^{-1}\right)\end{array}$ & $\begin{array}{l}K_{\mathrm{re}} \\
\left(\mathrm{J} \mathrm{m}^{-1} \mathrm{~s}^{-1} \mathrm{~K}^{-1}\right)\end{array}$ & $\begin{array}{l}\alpha_{\mathrm{w}} \\
\left(\mathrm{J} \mathrm{m}^{-2} \mathrm{~s}^{-1} \mathrm{~K}^{-1}\right)\end{array}$ \\
\hline 1 & 25.42 & 0.15 & 23.68 \\
2 & 22.18 & 0.15 & 15.90 \\
3 & 31.77 & 0.15 & 29.20 \\
4 & 27.73 & 0.15 & 27.90 \\
\hline
\end{tabular}

the wall where some discrepancies occur probably due to the flow maldistribution, an effect which is not considered in the modeling.

The parameters $P e_{\mathrm{h}}, P e_{\mathrm{r}}$ and $B i_{\mathrm{h}}$ were estimated by fitting the model solution to the experimental data and with their estimates the values of the thermal parameter $\left(K_{\mathrm{ae}}, K_{\mathrm{re}}\right.$ and $\left.\alpha_{\mathrm{w}}\right)$ shown in Table 2 were determined.

\section{Conclusions}

This work was conducted to study the heat transfer in a fixed-bed packed with polymeric adsorbent particles on the basis of experimental dynamic responses resulting from changes in the inlet fluid temperature.

A method based on the verification of the linear independence of sensitivity functions allowed to demonstrate that the thermal parameters could be estimated considering the experimental measurements obtained from the sensor located axially and radially in the column. Moreover, the method was also applied for determining optimal sensor location.

For the description of the experimental results the 1$\mathrm{D}$ and 2-D pseudo-homogeneous models were investigated. The 1-D model allowed a good dynamic representation of the fluid temperature at the outlet of the column from which the overall heat transfer coefficient and the axial Peclet number were estimated. The sensi- tivity behavior of the system at steady-state was also studied. Results show that the operating variables, namely, the flowrate and the bed height, and the overall heat transfer coefficient significantly influence the temperature profiles in the bed while the influence of the effective axial thermal conductivity can be ignored. Since the radial temperature can not be neglected, the 2-D model was developed to describe with greater confidence the experimental transient thermal responses. This model was capable of predicting such responses and with a suitable optimization algorithm (GREG software package [19]) the best estimates of the effective radial and axial conductivities and of the wall heat transfer coefficient were found. With regard to the sensitivity analysis, this model confirmed the results obtained previously with the 1-D model and led to the transient sensitivity functions of the parameters under investigation.

The methodology followed in this paper can be used as a good basis for the scale-up. However, it is necessary to know how the thermal parameters are influenced by the aspect ratio $R_{\mathrm{c}} / d_{\mathrm{p}}$ and the Reynolds number $R e$. So, changing to columns with larger diameter but keeping the same particle size the aspect ratio increases. Since for the system studied the aspect ratio is high $\left(R_{\mathrm{c}} / d_{\mathrm{p}} \approx 100\right)$ it is expectable that no significant influence occurs on the parameters when the column diameter increases. On the other hand, in columns of commercial size the flowrate is generally high which then will cause changes of $R e$ to higher values. The dependence of the effective radial conductivity and the wall heat transfer coefficient on number Reynolds can be analytically described by a linear function.

\section{Acknowledgements}

The authors would like to thank the assistance provided to the first author by Mrs. Maria J. P. Araújo, a fifth-year student in Chemical Engineering at the University of Coimbra.

\section{References}

[1] O.A. Asbjornsen, B. Wang, Heat transfer and diffusion in fixed beds, Chem. Eng. Sci. 26 (5) (1971) 585-604.

[2] A.G. Dixon, Thermal resistance models of packed-bed effective heat transfer parameters, AIChE J. 31 (5) (1985) 826-834.

[3] A.G. Dixon, An improved equation for the overall heat transfer coefficient in packed beds, Chem. Eng. Process. 35 (1996) 323-331.

[4] E.A. Foumeny, H. Pahlevanzadeh, Evaluation of plug flow assumption in packed beds, Chem. Eng. Technol. 13 (1990) 161-171. 
[5] E.A. Foumeny, C. McGreavy, H. Pahlevanzadeh, J.A.A Castro, A.E. Rodrigues, Estimation of transport coefficients in packed beds, IChem E. Symp. Ser. 129 (1992) 1077-1083.

[6] M. Chalbi, J.A. Castro, A.E. Rodrigues, A. Zoulalian, Heat transfer parameters in fixed bed exchangers, Chem. Eng. J. 34 (1987) 89-97.

[7] D.E. Beasley, J.A. Clark, Transient response of a packed bed for thermal energy storage, Int. J. Heat Mass Transfer 27 (9) (1984) 1659-1669.

[8] N. Point, A.V. Wouwer, M. Remy, Practical issues in distributed parameter estimation: gradient computation and optimal experiment design, Control Eng. Practice 4 (11) (1996) 1553-1562.

[9] H. Hwang, S. Chiao, Parameter sensitivity of temperature at the regeneration of a single catalyst pellet, Chem. Eng. Sci. 50 (4) (1995) 685-694.

[10] S. Ungureanu, C. Petrila, A. Mares, Elementary sensitivity of a chemical reactor described by a quasihomogeneous bidimensional model, Chem. Eng. Sci. 49 (7) (1994) 10151024.

[11] L.C. Windes, A. Cinar, W.H. Ray, Dynamic estimation of temperatures and concentration profiles in a packed bed reactor, Chem. Eng. Sci. 44 (10) (1989) 20872106.
[12] R. Yetter, L.A. Eslava, F.L. Dryer, H. Rabitz, Elementary and derived sensitivity information in chemical kinetics, J. Phys. Chem. 88 (1984) 1497-1507.

[13] R.J.V. Welsenaere, G.F. Froment, Parametric sensitivity and runway in fixed bed catalytic reactors, Chem. Eng. Sci. 25 (1970) 1503-1516.

[14] L. Ferreira, A.E. Rodrigues, Adsorptive separation by thermal parametric pumping, Adsorption J. 1 (1995) 213231.

[15] B.A. Finlayson, Non-Linear Analysis is Chemical Engineering, McGraw-Hill, New York, 1980.

[16] M. Caracotsios, W.E. Stewart, Sensitivity analysis of initial-boundary-value problems with mixed PDEs and algebraic equations: applications to chemical and biochemical systems, Comput. Chem. Eng. 19 (9) (1995) 1019-1030.

[17] J.J. Carberry, Chemical and Catalytic Reaction Engineering, McGraw-Hill, New York, 1976.

[18] J.E. Crider, A.S. Foss, Effective wall heat transfer coefficients and thermal resistances in mathematical models of packed beds, AIChE J. 11 (6) (1965) 1012-1019.

[19] W.E. Stewart, M. Caracotsios and J.P. Sørensen, Software Documentation of GREG - General Regression Software Package for NonLinear Parameter Estimation (Version of July 1993), University of Wisconsin at Madison, Madison, WI, 1993. 